\title{
EFEITO DA ROTAÇÃO DO QUADRIL NA SÍNDROME DA DOR FEMOROPATELAR
}

\author{
Gramani-Say K ${ }^{1}$, Pulzatto F ${ }^{1}$, Santos GM ${ }^{1}$, Vassimon-Barroso V ${ }^{1}$, Siriani de Oliveira A ${ }^{2}$, \\ Bevilaqua-Grossi D ${ }^{2}$, Monteiro-Pedro V ${ }^{1}$ \\ ${ }^{1}$ Laboratório de Avaliação e Intervenção em Ortopedia e Traumatologia, Departamento de Fisioterapia, Universidade \\ Federal de São Carlos, São Carlos, SP \\ ${ }^{2}$ Departamento de Biomecânica, Medicina e Reabilitação do Aparelho Locomotor, Faculdade de Medicina de Ribeirão \\ Preto, Universidade de São Paulo, Ribeirão Preto, SP \\ Correpondência para: Karina Gramani Say, Rua São José 409, Centro, CEP 14010-160, Ribeirão Preto, SP, e-mail: \\ kagramanis@yahoo.com.br
}

Recebido: 31/01/2005 - Aceito: 13/10/2005

\begin{abstract}
RESUMO
Objetivo: A proposta desse trabalho foi investigar o efeito da rotação do quadril na atividade elétrica dos músculos Vasto Medial Oblíquo (VMO), Vasto Lateral Oblíquo (VLO) e Vasto Lateral Longo (VLL) no agachamento a $45^{\circ}$ de flexão do joelho associado à posição neutra, rotação lateral a $45^{\circ}$ e rotação medial a 15 e $30^{\circ}$ de quadril. Materiais e Método: Foram avaliadas 27 mulheres,

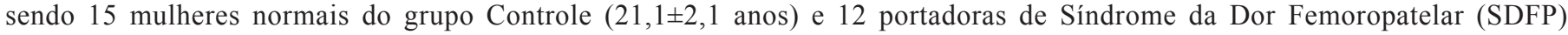
(21,0 $\pm 2,3$ anos). A atividade elétrica do músculo quadríceps foi captada por eletrodos ativos diferenciais simples de superfície, eletromiógrafo de 8 canais e programa Aqdados 7.2.6. Os dados foram normalizados pela porcentagem da média do agachamento a $75^{\circ}$ com o quadril em posição neutra - RMS $(\mu \mathrm{V})$. A análise estatística utilizada foi a ANOVA two way com medidas repetidas e Turkey post hoc $(\mathrm{p} \leq 0,05)$. Resultados: Os resultados evidenciaram interação entre grupos e músculos $(\mathrm{p}=0,00)$, independente da posição do quadril $(\mathrm{p}=0,39)$ e não houve interação entre grupos e posições do quadril $(\mathrm{p}=0,96)$. Os músculos VMO e VLL do grupo SDFP apresentaram atividade elétrica significativamente maior que o VMO $(p=0,00)$ e VLL ( $p=0,04)$ do grupo controle. Por outro lado, a atividade elétrica do músculo VLO não apresentou diferença significativa entre os grupos ( $\mathrm{p}=0,99)$. Conclusão: Os resultados dessa pesquisa, nas condições experimentais utilizadas, sugerem que a rotação do quadril não influenciou a atividade elétrica das porções do quadríceps nos dois grupos no agachamento a $45^{\circ}$ de flexão do joelho.
\end{abstract}

Palavras-chave: joelho, músculo quadríceps, síndrome da dor femoropatelar, eletromiografia, dor anterior no joelho.

\section{ABSTRACT \\ Effect of Hip Rotation on Patellofemoral Pain Syndrome}

Objective: The purpose of this study was to evaluate the effect of hip rotation on the electrical activity of the Vastus Medialis Obliquus (VMO), Vastus Lateralis Obliquus (VLO) and Vastus Lateralis Longus (VLL) muscles when squatting with $45^{\circ} \mathrm{knee}$ flexion, in association with the neutral position, $45^{\circ}$ lateral rotation and 15 and $30^{\circ}$ medial hip rotation. Method: 27 women were evaluated: 15 healthy women as a control group (21.1 \pm 2.1 years) and 12 subjects with patellofemoral pain syndrome (PFPS) $(21.0 \pm 2.3$ years $)$. The electrical activity of the quadriceps muscle was detected using simple active differential surface electrodes, an eight-channel electromyography system and the Aqdados 7.2.6 software. The data were normalized by the mean squatting percentage at $75^{\circ}$ with the hip in the neutral position - RMS $(\mu \mathrm{V})$. Two-way ANOVA with repeated measurements followed by the Tukey post hoc test $(\mathrm{p} \leq 0.05)$ was used for statistical analysis. Results: The results revealed interaction between groups and muscles $(\mathrm{p}=0.00)$, independent of hip position $(\mathrm{p}=0.39)$, and there was no interaction between groups and hip positions ( $\mathrm{p}=0.96)$. The VMO and VLL muscles of the PFPS group showed significantly greater electrical activity than did VMO ( $p=0.00)$ and VLL $(p=0.04)$ muscles from the control group. On the other hand, the electrical activity of the VLO muscle did not present any significant difference between the groups $(\mathrm{p}=0.99)$. Conclusion: Under the experimental conditions utilized, the results suggested that hip rotation did not alter the electrical activity of the quadriceps portions in either group when squatting with $45^{\circ} \mathrm{knee}$ flexion.

Key words: knee, quadriceps muscle, patellofemoral pain syndrome, electromyography, anterior knee pain. 


\section{INTRODUÇÃO}

A Síndrome da Dor Femoropatelar (SDFP) é definida como uma dor anterior ou retropatelar na ausência de outra patologia do joelho ${ }^{1}$, de etiologia multifatorial, sendo o maualinhamento patelar a hipótese mais freqüentemente aceita como o precursor primário de patologia da articulação Femoropatelar (FP) ${ }^{2}$. Considera-se atualmente, o maualinhamento do Membro Inferior, o desequilíbrio da musculatura extensora ${ }^{3}$, a insuficiência do $\mathrm{VMO}^{4}$, a fraqueza dos músculos do quadril ${ }^{5}$, a atividade excessiva, a diferença entre o início da contração muscular (onset) entre VMO e $\mathrm{VL}^{1}$ e a incongruência entre a patela e o sulco troclear femoral ${ }^{6}$ como fatores contribuintes para a origem da dor anterior no joelho.

Apesar da alta incidência, a etiologia da SDFP permanece indefinida, assim como o seu tratamento conservador ${ }^{3}$. Muitos autores têm sugerido os exercícios em Cadeia Cinética Fechada (CCF), como o agachamento e o "leg-press", por considerarem que esses exercícios reduzem a força de reação e o stress Femoropatelar ${ }^{7}$. Além disso, os exercícios realizados com o ângulo de $45^{\circ}$ são muito utilizados na prática clínica e a sua utilização é defendida em exercícios de CCF por ser considerada uma amplitude de movimento segura para a articulação femoropatelar e por ser o ângulo de maior congruência entre a patela e o sulco troclear ${ }^{7,8,9}$.

O tratamento conservador fisioterápico da SDFP tem como objetivo promover por meio de exercícios o equilíbrio entre as porções do músculo quadríceps ${ }^{8,10,11}$, especialmente os estabilizadores patelares mediais (Vasto Medial Oblíquo (VMO) e laterais (Vasto Lateral Oblíquo (VLO) e Vasto Lateral Longo (VLL) ${ }^{12,13}$. Dessa forma, vários autores recomendam posicionamentos diferentes do membro inferior com o intuito de melhorar a atividade muscular do VMO durante os exercícios em Cadeia Cinética Aberta (CCA $)^{10,14}$ e CCF no leg-press ${ }^{3,15}$ e nos exercícios de agachamento ${ }^{16,17}$. No entanto, não há um consenso sobre a eficácia dessas posições para o portador de $\operatorname{SDFP}^{18,19}$ e o saudável ${ }^{3,20}$.

O efeito da rotação do quadril na ativação do quadríceps ainda permanece controverso, uma vez que McConnel ${ }^{11}$ sugeriu o exercício de agachamento com rotação lateral de quadril (posição de plié) para o fortalecimento do VMO em indivíduos com dor por acreditar que essa posição facilitaria a ativação dessa porção muscular. Da mesma forma, Wheathley e Jahnke ${ }^{21}$ e Doucette e Goble ${ }^{22}$ afirmaram que ocorre uma ativação preferencial do Vasto Medial devido a um "stress" em valgo com a rotação lateral do quadril durante a extensão do joelho. No entanto, Lam e $\mathrm{Ng}^{19}$ relataram que a rotação medial do quadril favorece a ativação do VMO durante o agachamento em indivíduos com SDFP.

Os trabalhos que investigaram o efeito da rotação do quadril com o agachamento na ativação das porções do quadríceps, avaliaram o VMO e VLL em indivíduos com
$\mathrm{SDFP}^{18,19} \mathrm{e}$ em apenas um estudo foi verificada a atividade do VLO no agachamento no grupo SDFP assintomático ${ }^{23}$. Além disso, nenhum trabalho foi encontrado analisando o efeito da rotação do quadril nas três porções do quadríceps durante o agachamento, em indivíduos com SDFP sintomáticos e realizaram a comparação com um grupo controle.

Diante do exposto, a proposta desse trabalho foi investigar a atividade elétrica dos músculos VMO, VLL e VLO durante o exercício de agachamento isométrico "wall slide" a $45^{\circ}$ de flexão do joelho associado à posição neutra e a rotação de quadril, a $15^{\circ}$ e $30^{\circ}$ em medial e a $45^{\circ}$ em lateral, em indivíduos saudáveis e portadores de SDFP.

\section{MATERIAIS E MÉTODOS}

\section{Sujeitos}

Foram avaliadas 15 voluntárias saudáveis $(21,1 \pm 2,1$ anos; $54.4 \pm 8,6 \mathrm{Kg} ; 1,63 \pm 0,06 \mathrm{~m})$ e 12 portadoras de Síndrome da Dor Femoropatelar (SDFP) sintomáticas (21,0 $\pm 2,3$ anos; $58,8 \pm 6,5 \mathrm{Kg} ; 1,64 \pm 0,06 \mathrm{~m}$ ), do sexo feminino, sedentárias ou que não praticassem nenhuma atividade esportiva regularmente (mais de 2 vezes por semana). As voluntárias estudadas foram apenas do sexo feminino considerando as grandes diferenças biomecânicas entre os sexos e a maior incidência da disfunção nesse sexo ${ }^{6}$.

As voluntárias foram selecionadas por meio de uma avaliação física seguindo a ficha de Avaliação do Laboratório de Avaliação e Intervenção em Ortopedia e Traumatologia (LAIOT) e de acordo com os critérios de inclusão e exclusão do estudo assinaram o Termo de Consentimento Livre e Esclarecido para participação na pesquisa aprovada pelo Comitê de Ética em Pesquisa.

Para a inclusão no grupo de SDFP, a voluntária tinha que apresentar dor na articulação FP no último mês ${ }^{24} \mathrm{e}$ dor, em pelo menos 3 das seguintes atividades funcionais questionadas: agachar por tempo prolongado, subir ou descer escadas, ajoelhar, correr, permanecer muito tempo sentado, na contração isométrica do músculo quadríceps e durante a prática de esporte ${ }^{1,25}$, além de apresentar dor de intensidade pelo menos 2,0 cm na Escala Visual Analógica (EVA) na articulação FP na última semana ${ }^{24}$. Além disso, as voluntárias tinham que apresentar dor, registrada na EVA após a simulação de atividades como agachar a $90^{\circ}$ e descer um degrau de 25 $\mathrm{cm}$ de altura por 30 segundos $^{1,25}$ e apresentar pelo menos 3 dos sinais clínicos: Teste da Compressão da articulação FP; Crepitação patelar; ângulo Q superior a $16^{\circ}$; Pronação subtalar excessiva; Patela alta; Retração do trato iliotibial; Sensibilidade à palpação das facetas patelares; Torção tibial externa; Mau-alinhamento patelar - Patela medializada ou lateralizada; Presença de hipo ou hipermobilidade patelar e Sinal da Baioneta ${ }^{15,23}$. 
As voluntárias do grupo controle não apresentavam qualquer história de doença, lesão, trauma, cirurgia ou fratura de Membro Inferior, história de dor na articulação do joelho e presença de dor na articulação FP no último mês ${ }^{24}$, e poderiam apresentar dor, em apenas duas das atividades funcionais questionadas $^{1,25}$. Além disso, a voluntária saudável deveria assinalar dor 0 na EVA na última semana ${ }^{24}$ e não apresentar dor na simulação das atividades funcionais (agachar e descer um degrau de $25 \mathrm{~cm})^{1,25}$ e na palpação das facetas patelares.

Foram excluídos desse trabalho, tanto do grupo com SDFP como do controle, os indivíduos que apresentassem fratura prévia de ossos longos do Membro Inferior, cirurgia prévia no joelho, quadril ou tornozelo, subluxação patelar traumática aguda, derrame articular, lesões envolvendo as articulações de quadril ou tornozelo e dor na coluna lombar, uso de medicação e/ou fisioterapia prévia ao estudo ${ }^{18}$, além de sintomas por overuse (tendinites, bursite, ruptura articular ou tendínea) nos membros inferiores ou história de inflamação intermitente ou persistente na articulação do joelho durante 2 anos anteriores ${ }^{26}$, presença de outras lesões na articulação do joelho - meniscais, ligamentares ou capsulares e presença de doenças neurológicas ${ }^{18}$.

\section{Instrumentação}

Foi utilizado um Eletromiógrafo de 8 canais/ 12 bits de resolução (Emg System do Brasil Ltda), interfaciado com um microcomputador Pentium II e um programa para análise digital dos sinais, AqDados 7.2.6. Os sinais EMG foram coletados de forma sincrônica numa freqüência de amostragem de $2000 \mathrm{~Hz}$, com filtro passa alta de $20 \mathrm{~Hz}$ e passa baixa de $500 \mathrm{~Hz}$, com ganho de 50 vezes no eletromiógrafo e um Índice de Rejeição por Modulação Comum (IRMC) maior que $100 \mathrm{~dB}$.

Foram utilizados os eletrodos ativos diferenciais simples de superfície (EMG System do Brasil Ltda.) compostos por duas barras paralelas de $\mathrm{Ag}-\mathrm{AgCl}$, retangulares, cada uma com um $1 \mathrm{~cm}$ de comprimento, $1 \mathrm{~mm}$ de largura e distância de $1 \mathrm{~cm}$ entre si, envolvidas em uma cápsula de poliuretano contendo um microcircuito elétrico, que permitia que o sinal de EMG fosse pré-amplificado com ganho de 20 vezes para captação da atividade elétrica dos músculos. $\mathrm{O}$ eletrodo de referência tinha forma oval de 4,5 cm de comprimento e 3,0 $\mathrm{cm}$ de largura sendo utilizado para eliminar interferências externas $^{27}$. Os sinais foram quantificados em RMS (Root Mean Square) e expressos em microvolts $(\mu \mathrm{V})$.

A amplitude de flexão do joelho foi mensurada por meio de um eletrogoniômetro constituído de duas barras de madeira, cabos flexíveis de 2 metros de comprimento, fios blindados 30 AWG, com abertura de 0 a 225 graus, saída analógica de 0 a $5 \mathrm{~V}$, registro do sinal na unidade real de medida (em graus) e configurável em um dos canais do módulo condicionador.

O agachamento foi realizado sobre um dispositivo plano de madeira, denominado plataforma de agachamento, de 70 $\mathrm{cm}$ de largura por $50 \mathrm{~cm}$ de profundidade. A plataforma possuía dois aparatos para os pés reguláveis para o posicionamento adequado dos pés e quadril.

\section{Procedimentos}

Antes de iniciar a avaliação eletromiográfica, cada voluntária foi orientada e familiarizada com os procedimentos do estudo e foram instruídas a realizarem, previamente a avaliação de EMG, alongamento de 1 minuto para os músculos: quadríceps femoral, isquiotibiais e tríceps sural, com supervisão da fisioterapeuta.

A seqüência dos exercícios foi aleatória, por sorteio, definindo a ordem do posicionamento do membro inferior ${ }^{16}$. Durante todo o experimento a temperatura ambiente da sala foi mantida em cerca de $23^{\circ} \mathrm{C}$.

A pele da voluntária foi devidamente preparada no local da fixação dos eletrodos, sendo realizada a tricotomia, abrasão e limpeza com álcool 70\%, a fim de diminuir a sua impedância. Em seguida, foi traçada uma linha que unia a espinha ilíaca anterosuperior ao centro da patela, para referência das medidas dos ângulos de inclinação das porções musculares ${ }^{13}$, sendo os eletrodos fixados paralelamente ao alinhamento das fibras e os sítios de detecção perpendiculares a $\operatorname{estas}^{27}$.

O eletrodo de captação do sinal eletromiográfico do músculo VMO foi fixado a $4 \mathrm{~cm}$ da borda súpero-medial da patela $^{28}$, com um ângulo de $50-55^{\circ}$ de inclinação ${ }^{29}$, para o músculo VLL, o eletrodo foi fixado a $15 \mathrm{~cm}$ da borda súperolateral da patela e com $13^{\circ}$ de inclinação, no VLO foi fixado no ventre do músculo, entre o epicôndilo lateral e a junção miotendinosa, com inclinação de aproximadamente 50,4 ${ }^{\circ} 13$ e o eletrodo de referência foi fixado próximo à região maleolar homolateral do membro inferior avaliado ${ }^{17}$.

A voluntária era posicionada na plataforma de agachamento para realização do exercício de "wall slide”, com a região lombar apoiada numa bola suíça encostada na parede e os membros inferiores semiflexionados e afastados da parede. Foi também orientado a manter a cabeça ereta, cruzar os braços sobre o peito em direção ao ombro contralateral, mantendo assim um leve contato entre a bola suíça e as costas durante o agachamento. Essa postura tinha como objetivo permitir o máximo de descarga do peso corporal nos membros inferiores bilateralmente ${ }^{8}$.

A distância entre os pés foi igual à distância dos ombros do indivíduo, da borda lateral do acrômio direito até a borda lateral do acrômio esquerdo ${ }^{16}$, mensurada, por meio de fita métrica.

A voluntária flexionou lentamente o joelho até o ângulo avaliado, após o comando verbal: “Atenção! Prepare! Agache! Pare! Mantenha, Mantenha, Mantenha! Relaxe!”. Ao atingir o ângulo de flexão, a voluntária já com o pé posicionado no aparato, não realizou resistência para a rotação. A voluntária foi orientada a evitar realizar um valgo de joelhos durante a execução do exercício, principalmente durante a posição do membro inferior em rotação medial. 
As medidas de rotação do quadril, posição neutra, $15^{\circ}$ e $30^{\circ}$ de rotação medial e $45^{\circ}$ de rotação lateral, foram realizadas antes do início do exercício para avaliação eletromiográfica, sendo demarcadas por meio de fita adesiva na plataforma.

As voluntárias eram orientadas a manter a contração por 6 segundos em todos os exercícios. Cada exercício foi repetido 3 vezes, com intervalo de 1 minuto e de 2 minutos entre as modalidades de agachamento ${ }^{8,18}$.

\section{Normalização}

Para a normalização dos dados foram realizados dois procedimentos, o primeiro foi por meio da contração isométrica voluntária máxima (CIVM) de extensão do joelho à $90^{\circ}$ de flexão de joelho e quadril em CCA, numa mesa flexo-extensora com o indivíduo devidamente estabilizado ${ }^{23}$. O segundo procedimento foi o exercício de agachamento a $75^{\circ} \mathrm{com}$ o quadril na posição neutra. Os dados foram normalizados por esses dois procedimentos e foi verificado que o agachamento a $75^{\circ}$ apresentou o menor coeficiente de variação, o que indicou a maneira mais apropriada para normalizar os dados desse estudo. Além disso, Cowan et al. ${ }^{1}$ relataram que a CIVM em CCA não é a melhor forma para normalizar os dados eletromiográficos em indivíduos que apresentam dor, como nesse estudo, já que estes sujeitos não conseguem manter a contração isométrica máxima.

A média dos valores de RMS $(\mu \mathrm{V})$ obtida dos sinais eletromiográficos nos 3 exercícios de agachamento com associação da posição neutra e rotações medial e lateral do quadril foi dividida pelo valor da RMS da média do agachamento a $75^{\circ}$ de flexão do joelho e rotação neutra do quadril multiplicada por $100^{28}$.

Valor Médio de RMS dos exercícios de agachamento X 100 Valor Médio de RMS do agachamento a $75^{\circ} \mathrm{PN}$

\section{Análise Estatística}

O método estatístico empregado foi à análise de variância (ANOVA two way) com medidas repetidas considerando os fatores: grupo, posições do quadril. No caso de diferença estatística foi realizado o teste de Tukey post hoc, considerando $\mathrm{p} \leq 0.05$.

\section{RESULTADOS}

Os resultados evidenciaram diferença entre os músculos $(p=0,00)$, interação entre grupos e músculos $(p=0,001)$ independente da posição de quadril adotada. Não foi encontrada interação entre grupos e posições do quadril $(p=0,96)$ e não houve diferença significativa entre as posições do quadril $(\mathrm{p}=0,39)$.

O músculo VMO do grupo SDFP apresentou atividade elétrica significativamente maior que o músculo VMO do grupo controle $(p=0,000)$. Da mesma forma, o músculo VLL dos indivíduos com SDFP apresentou atividade elétrica significativamente maior que o músculo VLL do grupo controle $(p=0,04)$. Por outro lado, não houve diferença significativa entre a atividade elétrica do músculo VLO entre os dois grupos $(\mathrm{p}=0,99)$, independente da posição do quadril (Tabela $1)$.

Tabela 1. Médias e desvios-padrões dos registros de EMG (RMS) dos músculos VMO, VLO e VLL normalizados no agachamento com diferentes posições do quadril do grupo SDFP $(n=12)$ e grupo Controle $(n=15)$.

\begin{tabular}{ccccccc}
\hline \multicolumn{4}{c}{ GRUPO CONTROLE } & \multicolumn{3}{c}{ GRUPO SDFP } \\
\hline $45^{\circ} \mathrm{PN}$ & 37.83 & 38.33 & 47.57 & $49.15^{*}$ & 36.98 & $54.00^{* *}$ \\
& $( \pm 14.35)$ & $( \pm 17.77)$ & $( \pm 27.46)$ & $( \pm 18.72)$ & $( \pm 9.42)$ & $( \pm 10.82)$ \\
$45^{\circ} \mathrm{RL} 45$ & 44.61 & 42.71 & 54.40 & $54.86^{*}$ & 42.14 & $58.91^{* *}$ \\
& $( \pm 15.86)$ & $( \pm 21.03)$ & $( \pm 35.70)$ & $( \pm 18.60)$ & $( \pm 11.10)$ & $( \pm 13.60)$ \\
$45^{\circ} \mathrm{RM} 30$ & 34.84 & 33.52 & 43.59 & $49.68^{*}$ & 34.24 & $54.46^{* *}$ \\
& $( \pm 17.07)$ & $( \pm 15.63)$ & $( \pm 26.23)$ & $( \pm 19.97)$ & $( \pm 11.10)$ & $( \pm 15.90)$ \\
$45^{\circ} \mathrm{RM} 15$ & 38.67 & 37.30 & 47.78 & $46.72^{*}$ & 35.26 & $53.53^{* *}$ \\
& $( \pm 22.19)$ & $( \pm 21.96)$ & $( \pm 32.71)$ & $( \pm 19.89)$ & $( \pm 12.06)$ & $( \pm 16.81)$ \\
\hline
\end{tabular}

* Diferença em relação ao músculo VMO do grupo Controle $(\mathrm{p}=0,00)$. 


\section{DISCUSSÃO}

Neste trabalho, o exercício em CCF de agachamento foi escolhido por ser um movimento funcional presente nas atividades da vida diária, também muito utilizado nos programas de reabilitação por proporcionar maior estabilidade por meio da co-contração do músculo quadríceps e isquiotibiais, e propriocepção articular. Além disso, a descarga de peso facilita a ação do VMO nos exercícios em CCF comparando com exercícios em $\mathrm{CCA}^{30}$.

O presente estudo indicou que a rotação do quadril não influenciou a atividade elétrica do quadríceps e não favoreceu a ativação seletiva do músculo VMO, nos dois grupos, apesar da origem dessa porção nos adutores, mais especificamente nos músculos Adutor Magno e Adutor Longo, que embora não motores primários, também são rotadores mediais do quadril e poderiam fornecer uma maior estabilização com a rotação de quadril para a contração do músculo VMO. Considerando essa mesma relação anatômica, vários autores ${ }^{8,23,28}$ verificaram que exercícios associados com adução do quadril favoreciam a atividade elétrica do VMO.

Estes resultados não concordam com os de Lam e $\mathrm{Ng}^{19}$ que verificaram a influência da posição do quadril na atividade elétrica dos músculos VMO e VL no agachamento a $40^{\circ}$ de flexão do joelho associado à rotação medial do quadril a $30^{\circ}$ em indivíduos com SDFP. É importante ressaltar que os autores também analisaram o agachamento a $40^{\circ}$ de flexão do joelho associado a rotação lateral do quadril a $45^{\circ}$ e não encontraram diferença para a atividade elétrica das porções do quadríceps nos portadores de SDFP, assim como o presente estudo. A diferença nos resultados pode estar relacionada com o nível de dor dos indivíduos com SDFP, que no estudo de Lam e $\mathrm{Ng}^{19}$ foi estipulada em pelo menos $5 \mathrm{~cm}$ na EVA, enquanto no presente estudo foi considerado pelo menos $2 \mathrm{~cm}$, sugerindo que indivíduos com uma intensidade maior de dor seriam influenciados pelo posicionamento do quadril. Além disso, Lam e $\mathrm{Ng}^{19}$ não avaliaram um grupo controle.

A rotação medial do quadril não influenciou a atividade elétrica das porções do quadríceps em indivíduos com SDFP, nas duas angulações estudadas, ou seja, mesmo com a característica dos indivíduos com SDFP apresentarem um valgo do joelho em atividades de descarga de peso $^{31}$, devido a falta de controle da adução e rotação interna do quadril, essa característica não foi capaz de alterar a atividade elétrica durante o agachamento no grupo SDFP. Apesar de Lam e $\mathrm{Ng}^{19}$ defenderem o agachamento com rotação medial do quadril a $30^{\circ} \mathrm{em}$ portadores da SDFP, acreditamos que esse ângulo de rotação promove o posicionamento em valgo do joelho, aumentando o ângulo $\mathrm{Q}$ dinâmico e a tendência a força lateral na patela ${ }^{31}$. Analisamos um ângulo de rotação medial menor que $30^{\circ}$, ou seja, de $15^{\circ}$, para verificarmos se também encontraríamos uma maior atividade elétrica do VMO para o grupo SDFP; entretanto, em nenhuma das angulações de rotação medial encontramos alteração da atividade do quadríceps, nos dois grupos.

Os diferentes posicionamentos do membro inferior, tanto da tíbia quanto dos pés, visando o tratamento da SDFP já foram estudados, entretanto, Ninos et al. ${ }^{16}$ não encontraram diferença significativa na atividade muscular do VMO e VL, em indivíduos normais, nos exercícios isotônicos de agachamento com a rotação dos pés. Da mesma forma, Serrão et al. ${ }^{3}$ não evidenciaram influência da posição da tíbia (rotação medial, lateral e neutra) na atividade elétrica do VMO durante o exercício de extensão do joelho a $90^{\circ}$ no "leg-press" em indivíduos normais. No entanto, a alteração da posição da tíbia influenciou a atividade elétrica do VLL que foi maior na rotação medial em relação à posição neutra.

Os resultados mostram que o simples posicionamento em rotação do quadril não foi suficiente para influenciar a atividade do quadríceps sendo necessário verificar se a rotação resistida do quadril associada ao agachamento poderia alterar a atividade desses músculos. Apesar das diferenças metodológicas, Andrade et al. ${ }^{15}$ encontraram uma atividade elétrica maior do VMO em relação ao VLO tanto no grupo com SDFP como no Controle no exercício isométrico no "legpress" a $90^{\circ}$ de flexão do joelho e rotação isométrica lateral da tíbia, indicando essa rotação de Membro Inferior para o tratamento da SDFP.

No exercício de agachamento, o grupo SDFP apresentou atividade elétrica significativamente maior para os músculos VMO e VLL quando comparado com o grupo Controle, independente da posição do quadril. Entretanto, Coqueiro ${ }^{23}$ ao realizar o exercício de agachamento isolado e o agachamento associado à adução isométrica de quadril, ambos a $45^{\circ}$ de flexão do joelho, não encontraram diferença significativa entre o grupo SDFP e Controle para a atividade elétrica dos músculos VMO, VLO e VLL, o que mais uma vez sugere a importância da intensidade da dor visto que os indivíduos avaliados por Coqueiro ${ }^{23}$ eram assintomáticos.

Além disso, a maior atividade elétrica do músculo VMO e VLL do grupo SDFP em relação ao grupo Controle sugere que a causa da SDFP pode não estar relacionada à diminuição da atividade entre os componentes laterais e mediais do quadríceps, sendo necessário investigar outros possíveis parâmetros envolvidos com a dor na articulação femoropatelar como o início da ativação ("onset") entre as porções do quadríceps e a cinemática patelar por meio dos ângulos anatômicos (congruência, "tilt" patelar, sulco patelar e sulco troclear) da articulação femoropatelar. Laprade et al. ${ }^{18}$ sugeriram que uma atividade elétrica menor do grupo SDFP pode ocorrer secundariamente ao aparecimento da SDFP não sendo a função muscular o único fator etiológico da SDFP ao não encontrarem diferença na relação $\mathrm{VMO}$ : VL entre o grupo Controle e SDFP em exercícios de extensão do joelho associados à rotação medial dos pés e adução do quadril. 


\section{CONCLUSÃO}

Nas condições experimentais utilizadas, nossos dados indicam que a rotação do quadril não influencia a atividade elétrica do VMO, VLO e VLL, no ângulo de $45^{\circ}$ de agachamento. Os músculos VMO e VLL do grupo SDFP apresentaram atividade elétrica significativamente maior do que VMO e VLL do grupo Controle. Dessa forma, o exercício de agachamento à $45^{\circ}$ poderia ser indicado numa fase mais tardia da reabilitação de indivíduos com SDFP, uma vez que há um maior equilíbrio entre os estabilizadores da patela.

Agradecimentos: ao $\mathrm{CNPq}$ pelo apoio financeiro-Projeto Integrado de Pesquisa $n .^{\circ}$ 524190/96-8 e a CAPES/MEC pela bolsa concedida durante todo o período de realização desse trabalho. Ao Prof. Dr. Jorge Oishi pelo auxílio e orientação na análise estatística desse trabalho.

\section{REFERÊNCIAS BIBLIOGRÁFICAS}

1. Cowan SM, Bennell KL, Crossley KM, Hodges PW, Mcconnell J. Physical therapy alters recruitment of the vasti in patellofemoral pain syndrome. Medicine \& Science in Sports \& Exercise 2002; 1879-1885.

2. Powers CM. The influence of altered lower-extremity kinematics on patellofemoral joint dysfunction: A Theoretical perspective. Journal Orthopaedic \& Sports Physical Therapy 2003; 33(11): 639-646.

3. Serrão FV, Cabral CMN, Bérzin F, Monteiro-Pedro V. Effect of tibia rotation on the electromyographical activity of the vastus medialis oblique and vastus lateralis longus muscles during isometric leg-press. Physical Therapy In Sports 2005. in press.

4. Csintalan RP, Schults MM, Woo J, Mcmahon PJ, Lee TQ. Gender differences in patellofemoral joint biomechanics. Clinical Orthopaedic and related research 2002; 402: 260-269.

5. Ireland ML, Wilson JD, Ballantyne BT, Davis IM. Hip strength in females with and without patellofemoral pain. Journal Orthopaedic \& Sports Physical Therapy 2003; 33(11): 671676.

6. Powers CM. Patellar kinematics, part I: The influence of the Vastus Muscle activity in subjects with and without Patellofemoral Pain. Physical Therapy 2000; 80(10): 956-964.

7. Steinkamp LA. Biomechanical considerations in patellofemoral joint rehabilitation. Am. Journal Sports Medicine 1993; 21(3): 438-444.

8. Earl JE, Schmitz RJ, Arnold BL. Activation of the VMO and VL during dynamic mini-squat exercises with and without isometric hip adduction. Journal Electromyography and Kinesiology 2001; 11: 381-386.

9. Escamilla RF. Knee biomechanics of the dynamic squat exercise. Medicine \& Science in Sports \& Exercises 2001; 33(1): 127 141.
10. Cerny K. Vastus medialis oblique/vastus lateralis muscle activity ratios for selected exercises in persons with and without patellofemoral pain syndrome. Physical Therapy 1995; 75(8): 672-683.

11. Mcconnell J. Management of patellofemoral problems. Manual Therapy 1996; 1: 60-66.

12. Morrish GM, Woledge RC. A comparison of the activation of muscles moving the patella in normal subjects and in patients with chronic patellofemoral problems. Scandinavian Journal Rehabilitation Medicine 1997; 29: 43-48.

13. Bevilaqua-Grossi D, Monteiro-Pedro V, Bérzin F. Análise funcional dos estabilizadores da patela. Acta Ortopédica Brasileira 2004; 12(2): 99-104.

14. Mirzabeigi E, Jordan C, Gronley J, Perry J. Isolation of the Vastus Medialis Oblique Muscle during exercises. The American Journal Sports Medicine 1999; 27(1): 50-55.

15. Andrade PH, Bevilaqua-Grossi D, Bérzin F, Gil IA, MonteiroPedro V. Comparação da atividade elétrica do músculo Vasto Medial Oblíquo e Vasto Lateral Oblíquo em indivíduos com disfunção Femoropatelar. Revista de Fisioterapia da Universidade de São Paulo 2001; 8(2): 65-71.

16. Ninos JC, Irrgang JJ, Burdett R, Welss JR. Electromyographic analysis of the squat performed in self-selected lower extremity turn-out from the selected neutral position. Journal Orthopaedic \& Sports Physical Therapy 1997; 25(5): 307-315.

17. Blanpied P. Changes in muscles activation during wall slides and squat-machine exercise. Journal of Sports Rehabilitation 1999; 8: 123-134.

18. Laprade J, Culham E, Brouwer B. Comparison of five isometric exercises in the recruitment of the vastus medialis oblique in persons with and without patellofemoral pain syndrome. Journal Orthopaedic \& Sports Physical Therapy 1998; 27(3): 197-1-204.

19. Lam PL, Ng GYF. Activation of the quadriceps muscle during semisquatting with different hip and knee positions in patients with anterior knee pain. American Journal Physical Medicine and Rehabilitation 2001; 80(11): 804-808.

20. Signorile JF, Kacsik D, Perry A, Robertson B, Williams R. The effect of knee and foot position on the electromyographical activity of the superficial quadriceps. Journal Orthopaedic and Sports Physical Therapy 1995; 22(1): 29.

21. Wheathley M, Jahnke W. Electromyographical study of the superficial thigh and hip muscles in normal individuals. Archives Physical Medicine and Rehabilitation 1951; 32: 508-15.

22. Doucette SA, Goble EM. The effect of exercise on patellar tracking in lateral patellar compression syndrome. American Journal Sports Medicine 1992; 20(4): 434-440.

23. Coqueiro KRR.Exercício isométrico de agachamento associado à adução do quadril - análise elétrica dos músculos estabilizadores da patela. [Dissertação de Mestrado]. São Carlos (SP): Universidade Federal de São Carlos; 2003.

24. Cowan SM, Bennell KL, Hodges PW, Crossley KM, Mcconell J. Delayed onset of electromyographic activity of vastus medialis obliquus relative to vastus lateralis in subjects with patellofemoral pain syndrome. Archives of Physical Medicine and Rehabilitation 2001; 82(2): 183-189. 
25.Loudon JK, Wiesnert D, Goist-Foley HL, Asjes C, Loudon KL. Intrarater Reliability of functional performance tests for subjects with patellofemoral pain syndrome. Journal Athletic training. 2002; 73(3): 256-61.

26. Thomeé R. A comprehensive treatment approach for patellofemoral pain syndrome in young women. Physical Therapy 1997; 77(12): 1690-1703.

27. Deluca CJ. The use of surface electromyography in Biomechanics. Journal Applied Biomechanics 1997; 13: 135-163.

28. Hanten WP, Schulties SS. Exercises effect on electromyographic activity of the vastus medialis oblique and vastus lateralis muscles. Physical Therapy 1990; 70(9): 561-565.

29. Lieb FJ, Perry J. Quadriceps functions: an anatomical and mechanical study using amputed limbs. The Journal of Bone and Joint Surgery 1968; 53-A: 1535-1548.

30. Hodges PW, Richardson CA. The influence of isometric hip adduction on quadriceps femoris activity. Scandinavian Journal Rehabilitation 1993; 25: 57-62.

31. Mascal CL, Landel R, Powers CM. Management of Patellofemoral pain targeting hip, pelvis, and trunk muscle function: 2 casa reports. Journal Orthopaedic \& Sports Physical Therapy 2003, 33(11): 647-658. 